\title{
When Do Good Research Ideas Become Clinically Relevant
}

\author{
R. H. Belmaker ${ }^{1^{*}}$ \\ ${ }^{1}$ Visiting Prof. of Psychiatry at Hadassah-Hebrew University Medical Center, \\ Jerusalem, Israel.
}

*Corresponding to: R. H. Belmaker. Email: belmaker@bgu.ac.il.

I recently sat on a promotions committee at one of our universities regarding the promotion of a researcher who had had many brilliant ideas throughout his career that resulted in publications in high impact journals. His promotion, however, was complicated by several critical letters which pointed out that several of his ideas had turned out to be dead wrong, that he often seemed to continue to research his own ideas long after the consensus in science had turned to other areas of interest, and that he often defended his ideas with a passion that bordered on dogmatism. His promotion, although in a medical faculty, was unanimously approved because of the distinction between creative science and good medicine. Creative science of course involves many factors that we do not fully understand. However it is widely agreed that creative science involves intuition into the possibility of new truths that must be investigated with empirical methods. There is a time span during which the intuition remains merely an intuition and the hypothesis generates experiments. Because this experimental process takes time, effort and money, the conception of the hypothesis in science must be accompanied by a degree of enthusiasm and commitment that motivates the scientist to perform the experiments and achieve funding for his work, whether the hypothesis in the end turns out to be correct or not. Our laboratory spent several enthusiastic years studying the behavior of the inositol monophosphatase 1 (IMPA1) knockout mouse, which shows effects in the Porsolt mouse model of depression, before becoming aware of several dozen other gene knockouts that cause similar behavioral changes in the mouse ${ }^{[1]}$. We continued our work, but perhaps with somewhat more humility and less conviction of the kind that undeniably helps get grants and media attention.

In the medical treatment of a particular patient, however, the enthusiasm for an unproven belief that is so necessary in science can be terribly damaging. The clinician must plan his treatments on the basis of proven clinical facts, adapted to the circumstances of his individual patient. Hypothetical speculations are rarely helpful. New theories that inspire enthusiastic newspaper articles or even exciting symposia at international scientific conferences are rarely a good basis for a clinician to go home and change the treatment of individual patients. The 'incubation time' between hearing a new symposium in a new area whether it is the CRISPR technology for gene implantation or the microbiome effects on models of mood in rats, and the time when

Copyright: $\odot 2017$ Cain et al. This is an open access article distributed under the terms of the Creative Commons Attribution License, which permits unrestricted use, distribution, and reproduction in any medium, provided the original author and source are credited. 
it will be possible to recommend to our patients that they undergo gene therapy or fecal transplant is usually years, and of course often the ideas do not work out. The dangers of overselling to clinicians are clear, and regulatory agencies spend much of their effort answering patients, families and doctors who demand that a new treatment that has been shown recently in rats be available for treatment of their own illness. However, I believe that there is a more fundamental danger to overpromising and that is the erosion of long term support for science and medicine. We know several factors that are eroding the legitimacy of science in recent years:

1. The internet that allows a platform for so many unproven theories seems to provide a level playing field between the opinions of senior clinicians and scientists on the one side and kooks who may have no scientific and clinical experience and who find an audience in a platform on the internet, on the other side.

2. Contradictory scientific reports that may once have been discussed only in closed forums and are now known to the whole public such as the change in attitudes about cholesterol, blood pressure standards and hormone replacement therapy in post-menopausal women. For instance, evidence that antidepressants may induce mania in bipolar patients seemed to be the prevailing data in North America while European data sets concluded that this phenomenon does not exist ${ }^{[2]}$.

3. In addition to those, I think 'overpromising' has contributed also greatly in recent years to the erosion of the legitimacy of the scientific process. In order to write grants and get funding, all scientists have to explain the possible utility of their findings, even if these findings are pilot studies, first reports or proofs of principle.

The clinician is a person who can weigh the different evidence available today and decide and explain to his patient whether the exciting new finding is relevant for the patient's condition. For example, new theories and finding about the mechanism of lithium action have changed about every 10 years since lithium's discovery in 1950 and have come as part of paradigm shifts in the whole scientific zeitgeist ${ }^{[3]}$. Each paradigm shift led to new empirical findings that were highly publicized, were heuristic for scientific productivity and which seemed to provide a platform for rapid development for new anti-bipolar compounds. However none of these theories reached the stage where a clinician with a patient need take them into account. The explanatory process, whether to an individual patient or in the media, is critical to being a physician and to explaining medical thinking to the public. It is also important to avoid cynicism and the assumption that nothing in medicine will ever change and that none of the new treatments will work out. This is, of course, not true and we have seen major changes in all areas of medicine over the last 50 years. However, these changes are almost always slower than the changes depicted in our media, which have gone from weekly news magazines to daily newspapers to hourly radio reports to minute-by-minute Whatspps, not only in social areas but in scientific reporting as well. The doctor's commitment to judgement is an essence of the difference between a physician and a scientist, even though the two professions fertilize each other and sometimes coexist in the same individual who can wear both hats, but in different situations. One of my teachers used to emphasize at clinical rounds that the amount of scientific facts that a student could quote, irrelevant to the case at hand, were really of no use to the specific patient. Scientific evidence needs to be replicated first and then proven in controlled clinical trials before it is directly relevant to clinical care, except in rare and unusual cases.

For example, the possibility that BDNF is important for affective disorder and that lithium might work by increasing BDNF. This protein is clearly important in brain, involved in neuronal proliferation and neuroprotection, and affected by numerous psychiatric medicines including lithium. Scientific enthusiasm is great and progress of science should not be impeded by cynicism. However BDNF effects vary from species to species; treatments of neurodegenerative disease in animal models have almost always failed in human trials; BDNF as measured in peripheral blood in human studies seems to be present mostly in blood cells and is highly dependent on technical procedures that separate serum from plasma; peripheral BDNF may not be related to brain BDNF as peripheral endorphins are not related to brain endorphins, neural proliferation in humans seems to be less prominent that in some animal species. It is of little use to explain to a patient on the basis of these preliminary scientific reports that lithium will protect his brain from degeneration or will help him grow neurons. The clinician should base his explanation to patients on bodies of basic science knowledge that are widely replicable, have achieved a scientific coherence and are of true use in clinical decision making. Two controlled clinical trials, as demanded by the American FDA, is a good rule of thumb. 


\section{REFERENCES}

1. Belmaker RH, Agam G. Major depressive disorder. N Engl J Med. 2008; 358(1): 55-68.

2. Belmaker RH. Treatment of bipolar depression. N Engl J Med. 2007; 356(17): 1771-1773.

3. Belmaker RH. Bipolar disorder. N Engl J Med. 2004; 351(5): 476-486. 